\title{
Cerebrospinal Fluid Leak Presenting with Radiculitis and Delayed Onset Headache Following Intrathecal Pump Implantation
}

\author{
Laura Anne Lynem, MD, Saurabh Dang, MD, Amanda Trout, DO, Edgar Martinez, MD, and \\ Joseph Atallah, MD
}

The issue of cerebrospinal fluid (CSF) leak due to catheter-related failure persists despite new approaches to surgical techniques. There is limited literature about atypical presentations of post dural puncture headaches post intrathecal drug delivery system (IDDS) implantation. A 59-year-old woman with a past medical history of lumbar postlaminectomy syndrome, chronic pain, and lower back pain underwent an intrathecal drug delivery system implantation after exhausting other modalities for pain relief. The patient presented to the office one-month postoperatively with symptoms of lower back pain, left lower extremity radiculopathy, and lower back subcutaneous fluid collection. A dye study using catheter access port access revealed a patent intrathecal catheter with no fluid leakage or collection after injecting contrast dye through the catheter access port. A fine needle aspiration performed under ultrasound guidance revealed a clear fluid. Using laboratory evaluation, this was confirmed to be CSF using laboratory evaluation. An epidural blood patch was performed, which provided minimal radicular pain relief. Several days after, she began experiencing fronto-occipital headaches and the lumbar fluid collection reaccumulated. An intrathecal dye study again was done and showed retrograde flow from the catheter causing a lumbar fluid collection. A catheter exchange was performed resolving the CSF leak and the patient's symptoms.

Post dural puncture headaches after IDDS implantation may have an atypical presentation. Providers should ensure the intrathecal catheter is well secured and firmly anchored to prevent migration. Patient symptoms after IDDS implantation may necessitate further investigation, including: patient evaluation, confirming catheter continuity, and minimally invasive or surgical management. Complications from IDDS may be reduced with further research on equipment and implantation techniques.

Key words: Intrathecal drug delivery system, neuraxial analgesia, chronic pain, post dural puncture headache, post laminectomy syndrome, intrathecal catheters
Intrathecal drug delivery systems (IDDS) are indicated for neuropathic pain, visceral pain, pain syndromes, mixed nociceptive-neuropathic pain, spastic pain, cancer pain and pain not relived from other modalities such as oral, rectal, or transdermal opioid administration. IDDS provide pain relief by administering drugs around the central nervous system.

From : University of Toledo Medical Center, Toledo, $\mathrm{OH}$

Author for correspondence: Laura Anne Lynem, MD

Address: University of Toledo Medical Center, Department of Anesthesiology, 3000 Arlington Ave, Toledo, OH 43551

E-mail: la1706@gmail.com
Possible complications from IDDS include postdural puncture headache (PDPH), catheter-related problems, pump failures, infection, and adverse drug reactions (1). This case report presents a medically challenging case involving an atypical presentation of a PDPH that resulted in radicular symptoms and delayed onset of headaches post IDDS implantation.

\section{Case}

A 59-year-old woman with a past medical history of lumbar postlaminectomy syndrome and chronic lower back pain was evaluated for IDDS placement. After other modalities were exhausted for pain relief, she 
underwent an IDDS implantation. The patient presented to the office one month post operatively with lower back pain, left lower extremity radiculopathy, and lower back subcutaneous fluid collection. The patient's vital signs were stable. The lumbar fluid collection was $1.5 \mathrm{~cm} \times 1.5 \mathrm{~cm}$, fluctuant, and nontender to palpation. It had no visible discoloration, induration, or signs of infection. Neurological examination was within normal limits. The straight leg raising test was negative.

A dye study using catheter access port access revealed a patent intrathecal catheter with no fluid leakage or collection after injecting contrast dye through the catheter access port. A fine needle aspiration performed under ultrasound guidance revealed clear fluid. This was confirmed to be cerebrospinal fluid (CSF) using laboratory evaluation.

Since her symptoms had not resolved by the time of her one-week follow-up visit, a PDPH was suspected as the cause of her symptoms. An epidural blood patch was performed which provided minimal radicular pain relief. Several days after, she began experiencing fronto-occipital headaches and the lumbar fluid collection re-accumulated. An intrathecal dye study ruled out intrathecal catheter migration. However, retrograde flow was found along the paraspinal lumbar fascia tracking from the proximal catheter causing L3-L5 extradural fluid collection. A catheter exchange and reinforcement was performed resolving the CSF leak and the patient's symptoms.

\section{DISCUSSION}

One of the most common complications of IDDS insertion is a PDPH due to a dual defect from the introducer needle piercing through the dura (1). The issue of CSF leak due to catheter-related failure persists despite new approaches to surgical techniques (2). There could be a correlation between procedural factors and the incidence of PDPH. Some of the procedural factors include operator experience, the design of the needle, surgical orientation, and reinsertion of the stylet (2). The incidence of PDPH postimplantation of an IDDS is $23 \%$ (3). Of that $23 \%$, $79 \%$ were self-limiting with medical management and did not require further intervention. The remainder of patients with PDPH eventually require interventional procedures, such an epidural blood patch or fibrin glue, for relief; $88 \%$ of these patients experience full recovery after one treatment. Refractory cases may require surgical exploration or a repair (3).

Some debate concerning the efficacy of epidural blood patches exists in the literature with some studies citing as low as $21 \%$ relief (4). However, epidural blood patches are still an effective treatment for patients with refractory symptoms due to PDPH after IDDS implantation (5). Most providers believe patients have significant improvement of symptoms after only one treatment with an epidural blood patch but it may be necessary for patients to undergo a second epidural blood patch for complete resolution of symptoms (5). Placement of an effective epidural blood patch should be one level below the dural puncture site since the blood travels more cephalad than caudal (5). A study from Kawaguchi et al (6) suggests a computed tomography-guided or fluoroscopically guided epidural blood patch placement could be an option if a patient's symptoms persist despite a second blood patch. Abdulla et al (7) found the use of high volume prophylactic epidural blood patch during intrathecal catheter exchange may be adequate for preventing PDPH postoperatively $(7,8)$. Abdulla et al (7) also found that a survey conducted in the United States showed that $10 \%-31 \%$ of providers have applied prophylactic epidural blood patches for unintentional dural punctures or similar issues (7). A study conducted by Ackerman et al (9) compared obstetric patients who received prophylactic epidural blood patches versus controls who did not and found a high degree of success. Vasdev and Southern (10) found that placing an epidural blood patch less than 24 hours after an epidural placement has a $71 \%$ failure rate, but a $96 \%$ success rate if done after 24 hours. Their study included 5,705 epidural procedures with $1 \%$ of the cases developing a PDPH. Prophylactic epidural blood patches were performed on $13 \%$, but were only effective in $29 \%$ of the cases. It should be noted that blood bacterial infections are a potential adverse effect of prophylactic epidural blood patch procedures (10). Although there is limited information regarding the use of fibrin glue as an alternative to an epidural blood patch in treating a PDPH after IDDS implantation, a study from Freeman et al (11) reports success rates of $60 \%$ to $70 \%$. The possibility of autologous blood bacterial and viral meningeal 
infections from epidural blood patch procedure is a risk, especially in patients with coexisting infectious diseases. In a study from Atallah et al (12) a successful fibrin sealant was performed as an alternative to an epidural blood patch in a patient with co-existing infectious diseases who presented with a PDPH (12).

Postoperative IDDS catheter complications occurring immediately due to technically related issues include PDPH, CSF leakage, infection, catheter kinking, catheter occlusion, pump malfunction, wound dehiscence, and catheter migration (13). According to Follett and Naurmann (13) another common cause of IDDS complications are due to the provider's implantation technique. Procedure-related complications necessitate that providers utilize careful surgical techniques and follow strict IDDS implantation guidelines. The most common catheter-related IDDS implantation complication is catheter migration due to failure in anchoring the catheter to the underlying lumbar fascia (14). Albrecht et al (15) described a case of an accidental intraspinal catheter placement. They noted that catheter migration prevents the diffusion of medication into the CSF, preventing pain relief. Frequent neurological exams with IDDS refills, for example, may be helpful for early detection of catheter migration. Also, if providers suspect issues with catheter integrity, besides a thorough history and physical, an imaging study such as magnetic resonance imaging or computed tomography may be warranted (15).

Providers have adopted new techniques to prevent catheter dislodgment and minimize seroma formation, including suturing the catheter to the underlying lumbar fascia, using an anchoring device, and

\section{REFERENCES}

1. Harrington BE, Schmitt AM. Meningeal (postdural) puncture headache, unintentional dural puncture, and the epidural blood patch: A national survey of United States practice. Reg Anesth Pain Med 2009; 34:430-437.

2. Waise S, Gannon D. Reducing the incidence of post-dural puncture headache. Clin Med 2013; 13:32-34.

3. Neuman SA, Eldrige JS, Qu W, Freeman ED, Hoelzer BC. Post dural puncture headache following intrathecal drug delivery system placement. Pain Physician 2012; 16:101-107.

4. Rucklidge MW. All patients with a postdural puncture headache should receive an epidural blood patch. Int $J$ Obstet Anesth 2014; 23:171-174. applying a purse string suture (16). Another surgical approach to minimizing catheter migration is to place the distal IDDS catheter tip at the midpoint between the articular surfaces of the superior and inferior facet joints that are positioned between the 2 adjacent intervertebral foramina (17). However, these techniques have not reduced the incidence of either paraspinal catheter coiling or leakage at the catheter insertion site (18). Despite receiving a purse string suture, our patient developed a lumbar fluid collection and left lower extremity radiculopathy. A study conducted on patients with lower extremity radiculopathy found elevated levels of pro-inflammatory cytokines such as interleukins and tumor necrosis factor-alpha in their CSF (19). In particular, levels of interleukin 6 were doubled relative to controls, which could be a contributing factor to nerve root irritation in patients with radiculopathy. Complications from IDDS may be reduced with further research on equipment and implantation techniques (20).

\section{CONCLUSION}

Postdural puncture headaches after IDDS implantation may have an atypical presentation. Providers should ensure the intrathecal catheter is well secured and firmly anchored to prevent migration. Patient symptoms after IDDS implantation may necessitate further investigation, including: patient evaluation, confirming catheter continuity, or surgical management (18).

\section{Conflict of Interests}

We declare that there are no conflicts of interest regarding the publication of this paper.
5. Bendel MA, Moeschler SM, Qu W, Hanley E, Neuman SA, Eldrige JS, Hoelzer BC. Treatment of refractory postdural puncture headache after intrathecal drug delivery system implantation with epidural blood patch procedures: A 20-year experience. Pain Res Treat 2016; 2016:1-5.

6. Kawaguchi M, Hashizume K, Watanabe K, Inoue S, Furuya H. Fluoroscopically guided epidural blood patch in patients with postdural puncture headache after spinal and epidural anesthesia. J Anesth 2011; 25:450-453.

7. Abdulla S, Vielhaber S, Heinze HJ, Abdulla W. A new approach using high volume blood patch for prevention of post-dural puncture headache following intrathecal catheter pump exchange. Int J Crit IIIn Inj Sci 2015; 5:93-98. 
8. Gobin Lonjaret L, Pailhas A, Bayoumeu F, Minville V. Accidental dural puncture: Combination of prophylactic methods to avoid post-dural puncture headache. Ann Fr Anesth Reanim 2014; 33:95-97.

9. Ackerman WE, Juneja MM, Kaczorowski DM. Prophylactic epidural blood patch for the prevention of postdural puncture headache in the parturient. Anesthesiol Rev 1990; 17:45-49.

10. Vasdev GM, Southern P. A postdural puncture headache: The role of prophylactic epidural blood patch. Curr Pain Headache Rep 2001; 5:281-283.

11. Freeman ED, Hoelzer BC, Eldrige JS, Moeschler SM. Fibrin glue to treat spinal fluid leaks associated with intrathecal drug systems. Pain Pract 2014; 14:570-576.

12. Atallah J, Gage E, Koning J, Duggan J, Ramsey-Williams V, Scott S, Gaudin D, Sarhan M. Treatment of post-dural puncture headache using epidural injection of fibrin sealant as an alternative to autologous epidural blood patch (case report). Scand $J$ Pain 2014; 5:170-172.

13. Rauck RL. Intrathecal drug delivery. Semin Pain Med 2004; 2:27.

14. Follett KA, Naumann CP. A prospective study of catheter-related complications of intrathecal drug delivery systems. J Pain
Symptom Manage 2000; 19:209-215.

15. Albrecht E, Durrer A, Chédel D, Maeder P, Buchser E. Intraparenchymal migration of an intrathecal catheter three years after implantation. Pain 2005; 118:274-278.

16. Kamran S, Wright BD. Complications of intrathecal drug delivery systems. Neuromodulation 2001; 4:111-115.

17. Ko WM, Ferrante FM. New onset lumbar radicular pain after implantation of an intrathecal drug delivery system: Imaging catheter migration. Reg Anesth Pain Med 2006; 31:363-367.

18. Sparlin JA, de Leon-Casasola OA. Intrathecal pump implantation techniques. Tech Reg Anesth Pain Manag 2011; 15:158161.

19. Ohtori S, Suzuki M, Koshi T, Takaso M, Yamashita M, Inoue G, Eguchi Y, Kuniyoshi Y, Ochiai N, Kishida S, Nakamura J, Aoki Y, Ishikawa T, Arai G, Miyagi M, Kamoda H, Suzuki M, Toyone T, Takahashi KN. Proinflammatory cytokines in the cerebrospinal fluid of patients with lumbar radiculopathy. Eur Spine $J$ 2011; 20:942-946.

20. Kim JH, Nahm FS, Chang JE, Park SY, Kim YC, Lee SC. An intrathecally located broken catheter used for an intrathecal drug delivery system. J Korean Med Sci 2012; 27:1278-1281. 